\title{
Oscillation criteria of fractional differential equations
}

\author{
Da-Xue Chen
}

Correspondence: cdx2003@163. com

College of Science, Hunan Institute of Engineering, 88 East Fuxing Road, Xiangtan, Hunan 411104, P. R. China

\section{Abstract}

In this article, we are concerned with the oscillation of the fractional differential equation

$$
\left[r(t)\left(D_{-}^{\alpha} \gamma\right)^{\eta}(t)\right]^{\prime}-q(t) f\left(\int_{t}^{\infty}(v-t)^{-\alpha} \gamma(v) d v\right)=0 \text { for } t>0,
$$

where $D_{-}^{\alpha} y$ is the Liouville right-sided fractional derivative of order $\alpha \in(0,1)$ of $y$ and $\eta>0$ is a quotient of odd positive integers. We establish some oscillation criteria for the equation by using a generalized Riccati transformation technique and an inequality. Examples are shown to illustrate our main results. To the best of author's knowledge, nothing is known regarding the oscillatory behavior of the equation, so this article initiates the study.

MSC (2010): 34A08; 34C10.

Keywords: oscillation, fractional derivative, fractional differential equation

\section{Introduction}

The goal of this article is to obtain several oscillation theorems for the fractional differential equation

$$
\left[r(t)\left(D_{-}^{\alpha} \gamma\right)^{\eta}(t)\right]^{\prime}-q(t) f\left(\int_{t}^{\infty}(v-t)^{-\alpha} \gamma(v) d v\right)=0 \text { for } t>0,
$$

where $\alpha \in(0,1)$ is a constant, $\eta>0$ is a quotient of odd positive integers, $D_{-}^{\alpha} \gamma$ is the Liou-ville right-sided fractional derivative of order $\alpha$ of $y$ defined by $\left(D_{-}^{\alpha} \gamma\right)(t):=\frac{1}{\Gamma(1-\alpha)} \frac{d}{d t} \int_{t}^{\infty}(v-t)^{-\alpha} \gamma(v) d v$ for $t \in \mathbb{R}_{+}:=(0, \infty)$, here $\Gamma$ is the gamma function defined by $\Gamma(t):=\int_{0}^{\infty} v^{t-1} e^{-v} d v$ for $t \in \mathbb{R}_{+}$, and the following conditions are assumed to hold:

(A) $r$ and $q$ are positive continuous functions on $\left[t_{0}, \infty\right)$ for a certain $t_{0}>0$ and $f$ : $\mathbb{R} \rightarrow \mathbb{R}$ is a continuous function such that $f(u) /\left(u^{\eta}\right) \geq K$ for a certain constant $K>$ 0 and for all $u \neq 0$.

By a solution of (1.1) we mean a nontrivial function $y \in C\left(\mathbb{R}_{+}, \mathbb{R}\right)$ such that 
$\int_{t}^{\infty}(v-t)^{-\alpha} y(v) d v \in C^{1}\left(\mathbb{R}_{+}, \mathbb{R}\right), r(t)\left(D_{-}^{\alpha} \gamma\right)^{\eta}(t) \in C^{1}\left(\mathbb{R}_{+}, \mathbb{R}\right)$ and satisfying (1.1) for $t>0$. Our attention is restricted to those solutions of (1.1) which exist on $\mathbb{R}_{+}$and satisfy $\sup \left\{|y(t)|: t>t_{*}\right\}>0$ for any $t_{*} \geq 0$. A solution $y$ of (1.1) is said to be oscillatory if it is neither eventually positive nor eventually negative. Otherwise it is nonoscillatory. Equation (1.1) is said to be oscillatory if all its solutions are oscillatory.

The theory of fractional derivatives goes back to Leibniz's note in his list to L'Hospital [1], dated 30 September 1695, in which the meaning of the derivative of order $1 / 2$ is discussed. Leibniz's note led to the appearance of the theory of derivatives and integrals of arbitrary order, which by the end of nineteenth century took more or less finished form due primarily to Liouville, Grünwald, Letnikov, and Riemann. Recently, there have been several books on the subject of fractional derivatives and fractional integrals, such as the books [2-6].

For three centuries the theory of fractional derivatives developed mainly as a pure theoretical field of mathematics useful only for mathematicians. However, in the last few decades many authors pointed out that fractional derivatives and fractional integrals are very suitable for the description of properties of various real problems.

The mathematical modeling and simulation of systems and processes, based on the description of their properties in term of fractional derivatives, naturally leads to differential equations of fractional order and to the necessity to solve such equations. Fractional differential equations are generalizations of classical differential equations of integer order and have gained considerable popularity and importance during the past three decades or so, due mainly to their demonstrated applications in numerous seemingly diverse and widespread fields of science and engineering. Nowadays the number of scientific and engineering problems involving fractional calculus is already very large and still growing. It was found that various, especially interdisciplinary applications can be elegantly modeled with the help of the fractional derivatives. Fractional differentials and integrals provide more accurate models of systems under consideration. Some of the areas of present applications of fractional calculus include fluid flow, rheology, dynamical processes in self-similar and porous structures, diffusive transport Akin to diffusion, electrical networks, probability and statistics, control theory of dynamical systems, viscoelasticity, electrochemistry of corrosion, chemical physics, optics, and signal processing, economics, and so on; for example, see [7-12] and the references cited therein.

Many articles have investigated some aspects of fractional differential equations, such as the existence and uniqueness of solutions to Cauchy type problems, the methods for explicit and numerical solutions, and the stability of solutions, and we refer to [13-20]. However, to the best of author's knowledge very little is known regarding the oscillatory behavior of fractional differential equations up to now. In particular, nothing is known regarding the oscillation properties of (1.1) up to now. To develop the qualitative theory of fractional differential equations, it is of great interest to study the oscillation of (1.1). In this article, we establish several oscillation criteria for (1.1) by applying a generalized Riccati transformation technique and an inequality. Our results are essential new. We also provide several examples to illustrate the results. 


\section{Preliminaries and lemmas}

In this section, we present the definitions of fractional integrals and fractional derivatives, which are used throughout this article. More details can be found in [2-6]. We also give several lemmas, which are useful in establishing our results.

There are several kinds of definitions of fractional integrals and fractional derivatives, such as the Riemann-Liouville definition, the Caputo definition, the Liouville definition, the Grünwald-Letnikov definition, the Erdélyi-Kober definition and the Hadamard definition. We adopt the Liouville right-sided definition on the half-axis $\mathbb{R}_{+}$for the purpose of this article.

Definition 2.1 (Kilbas et al. [5]) The Liouville right-sided fractional integral of order $\beta>0$ of a function $g: \mathbb{R}_{+} \rightarrow \mathbb{R}$ on the half-axis $\mathbb{R}_{+}$is given by

$$
\left(I_{-g}^{\beta}\right)(t):=\frac{1}{\Gamma(\beta)} \int_{t}^{\infty}(v-t)^{\beta-1} g(v) d v \text { for } t>0,
$$

provided the right-hand side is pointwise defined on $\mathbb{R}_{+}$, where $\Gamma$ is the gamma function.

Definition 2.2 (Kilbas et al. [5]) The Liouville right-sided fractional derivative of order $\beta>0$ of a function $g: \mathbb{R}_{+} \rightarrow \mathbb{R}$ on the half-axis $\mathbb{R}_{+}$is given by

$$
\begin{aligned}
\left(D_{-g}^{\beta}\right)(t) & :=(-1)^{\lceil\beta\rceil} \frac{d^{\lceil\beta\rceil}}{d t^{\lceil\beta\rceil}}\left(I_{-}^{\lceil\beta\rceil-\beta} g\right)(t) \\
& =(-1)^{\lceil\beta\rceil} \frac{1}{\Gamma(\lceil\beta\rceil-\beta)} \frac{d^{\lceil\beta\rceil}}{d t^{\lceil\beta\rceil}} \int_{t}^{\infty}(v-t)^{\lceil\beta\rceil-\beta-1} g(v) d v \text { for } t>0,
\end{aligned}
$$

provided the right-hand side is pointwise defined on $\mathbb{R}_{+}$, where $\ulcorner\beta\rceil:=\min \{z \in \mathbb{Z}: z \geq \beta\}$ is the ceiling function.

Lemma 2.1 Let $y$ be a solution of (1.1) and

$$
G(t):=\int_{t}^{\infty}(v-t)^{-\alpha} y(v) d v \text { for } \alpha \in(0,1) \text { and } t>0,
$$

then

$$
G^{\prime}(t)=-\Gamma(1-\alpha)\left(D_{-}^{\alpha} \gamma\right)(t) \text { for } \alpha \in(0,1) \text { and } t>0 .
$$

Proof From (2.3) and (2.2), for $\alpha \in(0,1)$ and $t>0$ we obtain

$$
\begin{aligned}
G^{\prime}(t) & =\Gamma(1-\alpha) \frac{1}{\Gamma(1-\alpha)} \frac{d}{d t} \int_{t}^{\infty}(v-t)^{-\alpha} \gamma(v) d v \\
& =-\Gamma(1-\alpha)\left[(-1)^{\lceil\alpha\rceil} \frac{1}{\Gamma(\lceil\alpha\rceil-\alpha)} \frac{d^{\lceil\alpha\rceil}}{d t^{\lceil\alpha\rceil}} \int_{t}^{\infty}(v-t)^{\lceil\alpha\rceil-\alpha-1} \gamma(v) d v\right] \\
& =-\Gamma(1-\alpha)\left(D_{-}^{\alpha} \gamma\right)(t) .
\end{aligned}
$$

The proof is complete.

Lemma 2.2 (Hardy et al. [21]) If $X$ and $Y$ are nonnegative, then

$$
m X Y^{m-1}-X^{m} \leq(m-1) Y^{m} \text { for } m>1 \text {, }
$$


where the equality holds if and only if $X=Y$.

\section{Main results}

Theorem 3.1 Suppose that (A) and

$$
\int_{t_{0}}^{\infty} r^{-1 / \eta}(t) d t=\infty
$$

hold. Furthermore, assume that there exists a positive function $b \in C^{1}\left[t_{0}, \infty\right)$ such that

$$
\limsup _{t \rightarrow \infty} \int_{t_{0}}^{t}\left[K b(s) q(s)-\frac{r(s)\left[b^{\prime}+(s)\right]^{\eta+1}}{(\eta+1)^{\eta+1}[\Gamma(1-\alpha) b(s)]^{\eta}}\right] d s=\infty,
$$

where $b_{+}^{\prime}(s):=\max \left\{b^{\prime}(s), 0\right\}$. Then every solution of (1.1) is oscillatory.

Proof Suppose that $y$ is a nonoscillatory solution of (1.1). Without loss of generality, we may assume that $y$ is an eventually positive solution of (1.1). Then there exists $t_{1} \in$ $\left[t_{0}, \infty\right)$ such that

$$
y(t)>0 \text { and } G(t)>0 \text { for } t \in\left[t_{1}, \infty\right),
$$

where $G$ is defined as in (2.3). Therefore, it follows from (1.1) that

$$
\left[r(t)\left(D_{-}^{\alpha} \gamma\right)^{\eta}(t)\right]^{\prime}=q(t) f(G(t))>0 \text { for } t \in\left[t_{1}, \infty\right) .
$$

Thus $r(t)\left(D_{-}^{\alpha} \gamma\right)^{\eta}(t)$ is strictly increasing on $\left[t_{1}, \infty\right)$ and is eventually of one sign. Since $r(t)>0$ for $t \in\left[t_{0}, \infty\right)$ and $\eta>0$ is a quotient of odd positive integers, we see that $\left(D_{-}^{\alpha} \gamma\right)(t)$ is eventually of one sign. We now claim

$$
\left(D_{-}^{\alpha} y\right)(t)<0 \text { for } t \in\left[t_{1}, \infty\right) .
$$

If not, then $\left(D_{-}^{\alpha} \gamma\right)(t)$ is eventually positive and there exists $t_{2} \in\left[t_{1}, \infty\right)$ such that $\left(D_{-}^{\alpha} \gamma\right)\left(t_{2}\right)>0$. Since $r(t)\left(D_{-}^{\alpha} \gamma\right)^{\eta}(t)$ is strictly increasing on $\left[t_{1}, \infty\right)$, it is clear that $r(t)\left(D_{-}^{\alpha} \gamma\right)^{\eta}(t) \geq r\left(t_{2}\right)\left(D_{-}^{\alpha} \gamma\right)^{\eta}\left(t_{2}\right):=c_{1}>0$ for $t \in\left[t_{2}, \infty\right)$. Therefore, from (2.4) we have

$$
-\frac{G^{\prime}(t)}{\Gamma(1-\alpha)}=\left(D_{-}^{\alpha} \gamma\right)(t) \geq c_{1}^{1 / \eta_{r}-1 / \eta}(t) \text { for } t \in\left[t_{2}, \infty\right) .
$$

Integrating both sides of the last inequality from $t_{2}$ to $t$, we get

$$
\int_{t_{2}}^{t} r^{-1 / \eta}(s) d s \leq-\frac{G(t)-G\left(t_{2}\right)}{c_{1}^{1 / \eta} \Gamma(1-\alpha)}<\frac{G\left(t_{2}\right)}{c_{1}^{1 / \eta} \Gamma(1-\alpha)} \text { for } t \in\left[t_{2}, \infty\right) .
$$

Letting $t \rightarrow \infty$, we see $\int_{t_{2}}^{\infty} r^{-1 / \eta}(s) d s \leq \frac{G\left(t_{2}\right)}{c_{1}^{1 / \eta} \Gamma(1-\alpha)}<\infty$. This contradicts (3.1).

Hence, (3.5) holds. Define the function $w$ by the generalized Riccati substitution

$$
w(t)=b(t) \frac{-r(t)\left(D_{-}^{\alpha} y\right)^{\eta}(t)}{G^{\eta}(t)} \text { for } t \in\left[t_{1}, \infty\right) .
$$


Then we have $w(t)>0$ for $t \in\left[t_{1}, \infty\right)$. From (3.6), (1.1), (2.4), and (A), it follows that

$$
\begin{aligned}
w^{\prime}(t) & =b^{\prime}(t) \frac{-r(t)\left(D_{-}^{\alpha} \gamma\right)^{\eta}(t)}{G^{\eta}(t)}+b(t)\left[\frac{-r(t)\left(D_{-}^{\alpha} \gamma\right)^{\eta}(t)}{G^{\eta}(t)}\right]^{\prime} \\
& \leq b_{+}^{\prime}(t) \frac{-r(t)\left(D_{-}^{\alpha} \gamma\right)^{\eta}(t)}{G^{\eta}(t)}+b(t)\left[\frac{-\left[r(t)\left(D_{-}^{\alpha} \gamma\right)^{\eta}(t)\right]^{\prime}}{G^{\eta}(t)}+r(t)\left(D_{-}^{\alpha} \gamma\right)^{\eta}(t) \frac{\eta G^{\eta-1}(t) G^{\prime}(t)}{G^{2 \eta}(t)}\right] \\
& =b_{+}^{\prime}(t) \frac{w(t)}{b(\gamma)}+b(t)\left[\frac{-q(t) f(G(t))}{G^{\eta}(t)}+r(t)\left(D_{-}^{\alpha} \gamma\right)^{\eta}(t) \frac{\eta\left[-\Gamma(1-\alpha)\left(D_{-}^{\alpha} \gamma\right)(t)\right]}{G^{\eta+1}(t)}\right] \\
& \leq b_{+}^{\prime}(t) \frac{w(t)}{b(t)}-K b(t) q(t)-\eta \Gamma(1-\alpha) b(t) r(t)\left[\frac{w(t)}{b(t) r(t)}\right]^{1+1 / \eta} \\
& =-K b(t) q(t)+\frac{b_{+}^{\prime}(t)}{b(t)} w(t)-\eta \Gamma(1-\alpha)[b(t) r(t)]^{-1 / \eta} w^{1+1 / \eta}(t) \text { for } t \geq t_{1},
\end{aligned}
$$

where $b_{+}^{\prime}$ is defined as in Theorem 3.1. Taking

$$
m=1+\frac{1}{\eta}, \quad X=\frac{[\eta \Gamma(1-\alpha)]^{1 / m} w(t)}{[b(t) r(t)]^{1 /(\eta+1)}} \quad \text { and } \quad Y=\frac{\left[b_{+}^{\prime}(t) / b(t)\right]^{\eta}[b(t) r(t)]^{1 / m}}{m^{\eta}[\eta \Gamma(1-\alpha)]^{\eta / m}},
$$

from (3.7) and Lemma 2.2 we conclude that

$$
w^{\prime}(t) \leq-K b(t) q(t)+\frac{r(t)\left[b_{+}^{\prime}(t)\right]^{\eta+1}}{(\eta+1)^{\eta+1}[\Gamma(1-\alpha) b(t)]^{\eta}} \text { for } t \in\left[t_{1}, \infty\right) .
$$

Integrating both sides of the last inequality from $t_{1}$ to $t$, we obtain

$$
\int_{t_{1}}^{t}\left[K b(s) q(s)-\frac{r(s)\left[b_{+}^{\prime}(s)\right]^{\eta+1}}{(\eta+1)^{\eta+1}[\Gamma(1-\alpha) b(s)]^{\eta}}\right] d x \leq w\left(t_{1}\right)-w(t)<w\left(t_{1}\right) \text { for } t \in\left[t_{1}, \infty\right) .
$$

Letting $t \quad \rightarrow \quad \infty, \quad$ we $\quad$ get $\limsup _{t \rightarrow \infty} \int_{t_{1}}^{t}\left[K b(s) q(s)-\frac{r(s)\left[b_{+}^{\prime}(s)\right]^{\eta+1}}{(\eta+1)^{\eta+1}[\Gamma(1-\alpha) b(s)]^{\eta}}\right] d s \leq w\left(t_{1}\right)<\infty$, which contradicts (3.2). The proof is complete.

Theorem 3.2 Suppose that (A) and (3.1) hold. Furthermore, suppose that there exist a positive function $b \in C^{1}\left[t_{0}, \infty\right)$ and a function $H \in C(\mathbb{D}, \mathbb{R})$, where $\mathbb{D}:=\left\{(t, s): t \geq s \geq t_{0}\right\}$, such that

$$
H(t, t)=0 \text { for } t \geq t_{0}, H(t, s)>0 \text { for }(t, s) \in \mathbb{D}_{0},
$$

where $\mathbb{D}_{0}:=\left\{(t, s): t>s \geq t_{0}\right\}$, and $H$ has a nonpositive continuous partial derivative $\mathbb{D}_{0}$ on $\mathbb{D}_{0}$ with respect to the second variable and satisfies

$$
\limsup _{t \rightarrow \infty} \frac{1}{H\left(t, t_{0}\right)} \int_{t_{0}}^{t-1}\left[b(s) q(s) H(t, s)-\frac{b(s) r(s) h_{+}^{\eta}(t, s)}{K(\eta+1)^{\eta+1}[\Gamma(1-\alpha) H(t, s)]^{\eta}}\right] d s=\infty,
$$

where $h_{+}(t, s):=\max \left\{0, H_{s}^{\prime}(t, s)+H(t, s) \frac{b_{+}^{\prime}(s)}{b(s)}\right\}$ for $(t, s) \in \mathbb{D}_{0}$, here $b_{+}^{\prime}$ is defined as in Theorem 3.1. Then all solutions of (1.1) are oscillatory.

Proof Suppose that $y$ is a nonoscillatory solution of (1.1). Without loss of generality, we may assume that $y$ is an eventually positive solution of (1.1). We proceed as in the 
proof of Theorem 3.1 to get that (3.7) holds. Multiplying (3.7) by $H(t, s)$ and integrating from $t_{1}$ to $t-1$, for $t \in\left[t_{1}+1, \infty\right)$ we obtain

$$
\begin{aligned}
\int_{t_{1}}^{t-1} K b(s) q(s) H(t, s) d s \leq & -\int_{t_{1}}^{t-1} H(t, s) w^{\prime}(s) d s+\int_{t_{1}}^{t-1} H(t, s) \frac{b_{+}^{\prime}(s)}{b(s)} w(s) d s \\
& -\int_{t_{1}}^{t-1} H(t, s) \eta \Gamma(1-\alpha)[b(s) r(s)]^{-1 / \eta} w^{1+1 / \eta}(s) d s .
\end{aligned}
$$

Using the integration by parts formula, for $t \in\left[t_{1}+1, \infty\right)$ we get

$$
\begin{aligned}
-\int_{t_{1}}^{t-1} H(t, s) w^{\prime}(s) d s & =-[-H(t, s) w(s)]_{s=t_{1}}^{s=t-1}+\int_{t_{1}}^{t-1} H_{s}^{\prime}(t, s) w(s) d s \\
& <H\left(t, t_{1}\right) w\left(t_{1}\right)+\int_{t_{1}}^{t-1} H_{s}^{\prime}(t, s) w(s) d s .
\end{aligned}
$$

Substituting (3.10) in (3.9), for $t \in\left[t_{1}+1, \infty\right)$ we have

$$
\begin{aligned}
& K \int_{t_{1}}^{t-1} b(s) q(s) H(t, s) d s \\
& \quad \leq H\left(t, t_{1}\right) w\left(t_{1}\right)+\int_{t_{1}}^{t-1}\left\{\left[H_{s}^{\prime}(t, s)+H(t, s) \frac{b_{+}^{\prime}(s)}{b(s)}\right] w(s)-\frac{\eta \Gamma(1-\alpha) H(t, s)}{[b(s) r(s)]^{1 / \eta}} w^{1+1 / \eta}(s)\right\} d s \\
& \quad \leq H\left(t, t_{1}\right) w\left(t_{1}\right)+\int_{t_{1}}^{t-1}\left[h_{+}(t, s) w(s)-\frac{\eta \Gamma(1-\alpha) H(t, s)}{[b(s) r(s)]^{1 / \eta}} w^{1+1 / \eta}(s)\right] d s,
\end{aligned}
$$

where $h_{+}$is defined as in Theorem 3.2. Taking

$$
m=1+\frac{1}{\eta}, \quad X=\frac{[\eta \Gamma(1-\alpha) H(t, s)]^{1 / m} w(s)}{[b(s) r(s)]^{1 /(\eta+1)}} \text { and } Y=\frac{h_{+}^{\eta}(t, s)[b(s) r(s)]^{1 / m}}{m^{\eta}[\eta \Gamma(1-\alpha) H(t, s)]^{\eta / m}}
$$

by using Lemma 2.2 in (3.11) we obtain for $t \in\left[t_{1}+1, \infty\right)$,

$$
\int_{t_{1}}^{t-1} b(s) q(s) H(t, s) d s \leq K^{-1} H\left(t, t_{1}\right) w\left(t_{1}\right)+K^{-1} \int_{t_{1}}^{t-1} \frac{b(s) r(s) h_{+}^{\eta}(t, s)}{(\eta+1)^{\eta+1}[\Gamma(1-\alpha) H(r, s)]^{\eta}} d s .
$$

Since $H_{s}^{\prime}(t, s) \leq 0$ for $t>s \geq t_{0}$, we have $0>H\left(t, t_{1}\right) \leq H\left(t, t_{0}\right)$ for $t>t_{1} \geq t_{0}$. Therefore, from (3.12) we get for $t \in\left[t_{1}+1, \infty\right)$,

$$
\begin{aligned}
& \int_{t_{1}}^{t-1}\left[b(s) q(s) H(t, s)-\frac{b(s) r(s) h_{+}^{\eta}(t, s)}{K(\eta+1)^{\eta+1}[\Gamma(1-\alpha) H(t, s)]^{\eta}}\right] d s \\
& \quad \leq K^{-1} H\left(t, t_{1}\right) w\left(t_{1}\right) \leq K^{-1} H\left(t, t_{0}\right) w\left(t_{1}\right) .
\end{aligned}
$$

Since $0<H(t, s) \leq H\left(t, t_{0}\right)$ for $t>s \geq t_{0}$, we have $0<\frac{H(t, s)}{H\left(t, t_{0}\right)} \leq 1$ for $t>s \geq t_{0}$. Hence, it follows from (3.13) that 


$$
\begin{aligned}
& \frac{1}{H\left(t_{,} t_{0}\right)} \int_{t_{0}}^{t-1}\left[b(s) q(s) H(t, s)-\frac{b(s) r(s) h_{+}^{\eta}(t, s)}{K(\eta+1)^{\eta+1}[\Gamma(1-\alpha) H(t, s)]^{\eta}}\right] d s \\
& =\frac{1}{H\left(t, t_{0}\right)} \int_{t_{0}}^{t_{1}}\left[b(s) q(s) H(t, s)-\frac{b(s) r(s) h_{+}^{\eta}(t, s)}{K(\eta+1)^{\eta+1}[\Gamma(1-\alpha) H(t, s)]^{\eta}}\right] d s \\
& \quad+\frac{1}{H\left(t, t_{0}\right)} \int_{t_{1}}^{t-1}\left[b(s) q(s) H(t, s)-\frac{b(s) r(s) h_{+}^{\eta}(t, s)}{K(\eta+1)^{\eta+1}[\Gamma(1-\alpha) H(t, s)]^{\eta}}\right] d s \\
& \leq \frac{1}{H\left(t, t_{0}\right)} \int_{t_{0}}^{t_{1}} b(s) q(s) H(t, s) d s+\frac{1}{H\left(t, t_{0}\right)} K^{-1} H\left(t, t_{0}\right) w\left(t_{1}\right) \\
& \leq \int_{t_{0}}^{t_{1}} b(s) q(s) d s+K^{-1} w\left(t_{1}\right) \text { for } t \in\left[t_{1}+1, \infty\right) .
\end{aligned}
$$

Letting $t \rightarrow \infty$, we have

$$
\begin{aligned}
& \limsup _{t \rightarrow \infty} \frac{1}{H\left(t, t_{0}\right)} \int_{t_{0}}^{t-1}\left[b(s) q(s) H(t, s)-\frac{b(s) r(s) h_{+}^{\eta}(t, s)}{K(\eta+1)^{\eta+1}[\Gamma(1-\alpha) H(t, s)]^{\eta}}\right] d s \\
& \leq \int_{t_{0}}^{t_{1}} b(s) q(s) d s+K^{-1} w\left(t_{1}\right)<\infty,
\end{aligned}
$$

which is a contradiction to (3.8). The proof is complete.

Next, we consider the case

$$
\int_{t_{0}}^{\infty} r^{-1 / \eta}(t) d t<\infty
$$

which yields (3.1) doesn't hold. In this case, we have the following results.

Theorem 3.3 Suppose that (A) and (3.14) hold and that there exists a positive function $b \in C^{1}\left[t_{0}, \infty\right)$ such that (3.2) holds. Furthermore, assume that for every constant $C$ $\geq t_{0}$

$$
\int_{C}^{\infty}\left[\frac{1}{r(t)} \int_{C}^{t} q(s) d s\right]^{1 / \eta} d t=\infty .
$$

Then every solution $y$ of (1.1) is oscillatory or satisfies $\lim _{t \rightarrow \infty} \int_{t}^{\infty}(v-t)^{-\alpha} \gamma(v) d v=0$. Proof Assume that $y$ is a nonoscillatory solution of (1.1). Without loss of generality, assume that $y$ is an eventually positive solution of (1.1). Proceeding as in the proof of Theorem 3.1, we obtain that (3.3) and (3.4) hold. Then there are two cases for the sign of $\left(D_{-}^{\alpha} \gamma\right)(t)$. The proof when $\left(D_{-}^{\alpha} \gamma\right)(t)$ is eventually negative is similar to that of Theorem 3.1 and hence is omitted.

Next, assume that $\left(D_{-\gamma}^{\alpha} \gamma\right)(t)$ is eventually positive. Then there exists $t_{2} \geq t_{1}$ such that $\left(D_{-}^{\alpha} \gamma\right)(t)>0$ for $t \geq t_{2}$. From (2.4) we get $G^{\prime}(t)<0$ for $t \geq t_{2}$. Thus we get $\lim _{t \rightarrow \infty} G(t)$ $:=L \geq 0$ and $G(t) \geq L$. We now claim $L=0$. Assume not, i.e., $L>0$, then from (3.4) 
we get

$$
\left[r(t)\left(D_{-}^{\alpha} \gamma\right)^{\eta}(t)\right]^{\prime}=q(t) f(G(t)) \geq q(t) K G^{\eta}(t) \geq K L^{\eta} q(t) \text { for } t \in\left[t_{2}, \infty\right) .
$$

Integrating both sides of the last inequality from $t_{2}$ to $t$, we have

$$
r(t)\left(D_{-}^{\alpha} \gamma\right)^{\eta}(t) \geq r\left(t_{2}\right)\left(D_{-}^{\alpha} \gamma\right)^{\eta}\left(t_{2}\right)+K L^{\eta} \int_{t_{2}}^{t} q(s) d s>K L^{\eta} \int_{t_{2}}^{t} q(s) d s \text { for } t \in\left[t_{2}, \infty\right) .
$$

Hence, from (2.4) we get

$$
-\frac{G^{\prime}(t)}{\Gamma(1-\alpha)}=\left(D_{-}^{\alpha} \gamma\right)(t)>K^{1 / \eta} L\left[\frac{1}{r(t)} \int_{t_{2}}^{t} q(s) d s\right]^{1 / \eta} \text { for } t \in\left[t_{2}, \infty\right) .
$$

Integrating both sides of the last inequality from $t_{2}$ to $t$, we obtain

$$
G(t) \leq G\left(t_{2}\right)-\Gamma(1-\alpha) K^{1 / \eta} L \int_{t_{2}}^{t}\left[\frac{1}{r(u)} \int_{t_{2}}^{u} q(s) d s\right]^{1 / \eta} d u \text { for } t \in\left[t_{2}, \infty\right) .
$$

Letting $t \rightarrow \infty$, from (3.15) we get $\lim _{t \rightarrow \infty} G(t)=-\infty$. This contradicts (3.3). Therefore, we have $L=0$, i.e., $\lim _{t \rightarrow \infty} G(t)=0$. In view of (2.3), we see that the proof is complete.

Theorem 3.4 Suppose that (A) and (3.14) hold. Let $b(t)$ and $H(t, s)$ be defined as in Theorem 3.2 such that (3.8) holds. Furthermore, assume that for every constant $C \geq t_{0}$, (3.15) holds. Then every solution $y$ of (1.1) is oscillatory or satisfies $\lim _{t \rightarrow \infty} \int_{t}^{\infty}(v-t)^{-\alpha} y(v) d v=0$.

Proof Assume that $y$ is a nonoscillatory solution of (1.1). Without loss of generality, assume that $y$ is an eventually positive solution of (1.1). Proceeding as in the proof of Theorem 3.1, we obtain that (3.3) and (3.4) hold. Then there are two cases for the sign of $\left(D_{-}^{\alpha} \gamma\right)(t)$. The proof when $\left(D_{-}^{\alpha} \gamma\right)(t)$ is eventually negative is similar to that of Theorem 3.2 and hence is omitted. The proof when $\left(D_{-}^{\alpha} \gamma\right)(t)$ is eventually positive is similar to that of the proof of Theorem 3.3 and thus is omitted. The proof is complete.

Remark 3.1 From Theorems 3.1-3.4, we can derive many different sufficient conditions for the oscillation of (1.1) with different choices of the functions $b$ and $H$.

\section{Examples}

Example 4.1 Consider the fractional differential equation

$$
\left[t^{\eta-1}\left(D_{-}^{\alpha} \gamma\right)^{\eta}(t)\right]^{\prime}-\frac{1}{t^{2}}\left(\int_{t}^{\infty}(v-t)^{-\alpha} \gamma(v) d v\right)^{\eta}=0, \quad t>0,
$$

where $\alpha \in(0,1), \eta>0$ is a quotient of odd positive integers and $(\eta+1)^{\eta+1}[\Gamma(1-\alpha)]$ $\eta>1$. In (4.1), $r(t)=t^{\eta-1}, q(t)=\frac{1}{t^{2}}$ and $f(u)=u^{\eta}$. Take $t_{0}>0$ and $K=1$. Since

$$
\int_{t_{0}}^{\infty} r^{-1 / \eta}(t) d t=\int_{t_{0}}^{\infty} \frac{1}{t^{1-\frac{1}{\eta}}} d t=\infty
$$


we find that (A) and (3.1) hold. We will apply Theorem 3.1, and it remains to satisfy the condition (3.2). Taking $b(s)=s$, we obtain

$$
\begin{aligned}
& \limsup _{t \rightarrow \infty} \int_{t_{0}}^{t}\left[K b(s) q(s)-\frac{r(s)\left[b_{+}^{\prime}(s)\right]^{\eta+1}}{(\eta+1)^{\eta+1}[\Gamma(1-\alpha) b(s)]^{\eta}}\right] d s \\
& \quad=\limsup _{t \rightarrow \infty} \int_{t_{0}}^{t} \frac{1}{s}\left[1-\frac{1}{(\eta+1)^{\eta+1}[\Gamma(1-\alpha)]^{\eta}}\right] d s \\
& \quad=\infty
\end{aligned}
$$

which implies that (3.2) holds. Therefore, by Theorem 3.1 every solution of (4.1) is oscillatory.

Example 4.2 Consider the fractional differential equation

$$
\left[t^{\eta+1}\left(D_{-}^{\alpha} \gamma\right)^{\eta}(t)\right]^{\prime}-2 t\left[3+\exp \left(\int_{t}^{\infty}(v-t)^{-\alpha} \gamma(v) d v\right)\right]\left(\int_{t}^{\infty}(v-t)^{-\alpha} \gamma(v) d v\right)^{\eta}=0, \quad t>0,
$$

where $\alpha \in(0,1)$ and $\eta>0$ is a quotient of odd positive integers. In (4.2), $r(t)=t^{\eta+1}$, $q(t)=2 t$ and $f(u)=\left(3+e^{u}\right) u^{\eta}$. Take $t_{0}>0$ and $K=3$. Since

$$
\int_{t_{0}}^{\infty} r^{-1 / \eta}(t) d t=\int_{t_{0}}^{\infty} \frac{1}{t^{1+\frac{1}{\eta}}} d t<\infty
$$

we see that (A) and (3.14) hold 3.3, it remains. To apply Theorem to satisfy the conditions (3.2) and (3.15). Take $b(s)=1$, then we get

$$
\limsup _{t \rightarrow \infty} \int_{t_{0}}^{t}\left[K b(s) q(s)-\frac{r(s)\left[b_{+}^{\prime}(s)\right]^{\eta+1}}{(\eta+1)^{\eta+1}[\Gamma(1-\alpha) b(s)]^{\eta}}\right] d s=\limsup _{t \rightarrow \infty} \int_{t_{0}}^{t} 6 s d s=\infty
$$

which implies that (3.2) holds. For every constant $C \geq t_{0}$, we can find $0<M<1$ and $t_{M} \geq C$ such that $t-C \geq M t$ for $t \in\left[t_{M}, \infty\right)$. Thus, we conclude

$$
\begin{aligned}
\int_{C}^{\infty} & {\left[\frac{1}{r(t)} \int_{C}^{t} q(s) d s\right]^{1 / \eta} d t=\int_{C}^{\infty}\left[\frac{1}{t^{\eta+1}} \int_{C}^{t} 2 s d s\right]^{1 / \eta} d t } \\
& =\int_{C}^{\infty}\left(\frac{t^{2}-C^{2}}{t^{\eta+1}}\right)^{1 / \eta} d t=\int_{C}^{\infty}\left[\frac{(t+C)(t-C)}{t^{\eta+1}}\right]^{1 / \eta} d t \\
& \geq(2 C M)^{\frac{1}{\eta}} \int_{t_{M}}^{\infty} \frac{1}{t} d t=\infty
\end{aligned}
$$

which yields that (3.15) holds. Hence, by Theorem 3.3 every solution $y$ of (4.2) is oscillatory or satisfies $\lim _{t \rightarrow \infty} \int_{t}^{\infty}(v-t)^{-\alpha} \gamma(v) d v=0$.

\section{Acknowledgements}

This study was supported by Hunan Provincial Natural Science Foundation of P. R. China (Grant No. 11JJ3010) and by the Science and Technology Program of Hunan Province of P. R. China (Grant No. 2010FJ6021). D.-X. Chen wish to thank the anonymous referees for their valuable suggestions and comments, which helped the author to improve the previous manuscript of the article. 


\section{Competing interests}

The author declares that he has no competing interests.

Received: 27 November 2011 Accepted: 15 March 2012 Published: 15 March 2012

\section{References}

1. Leibniz, GW: Mathmatische Schiften. Grorg Olms Verlagsbuchhandlung, Hildesheim (1962)

2. Oldham, KB, Spanier, J: The Fractional Calculus. Academic Press, San Diego (1974)

3. Miller, KS, Ross, B: An Introduction to The Fractional Calculus and Fractional Differential Equations. John Wiley \& Sons, New York (1993)

4. Podlubny, l: Fractional Differential Equations. Academic Press, San Diego (1999)

5. Kilbas, AA, Srivastava, HM, Trujillo, JJ: Theory and Applications of Fractional Differential Equations. Elsevier Science BV, Amsterdam (2006)

6. Das, S: Functional Fractional Calculus for System Identification and Controls. Springer, New York (2008)

7. Bagley, RL, Torvik, PJ: A theoretical basis for the application of fractional calculus to viscoelasticity. J Rheol. 27, 201-210 (1983). doi:10.1122/1.549724

8. Mainardi, F: Fractional calculus: Some basic problems in continuum and statistical mechanics. In: Carpinteri, A, Mainardi, F (eds.) Fractals and Fractional Calculus in Continuum Mechanics. pp. 291-348. Springer, New York (1997)

9. Mandelbrot, B: Some noises with 1/f spectrum, a bridge between direct current and white noise. IEEE Trans Inf Theory. 13, 289-298 (1967)

10. Rossikhin, YA, Shitikova, MV: Applications of fractional calculus to dynamic problems of linear and nonlinear hereditary mechanics of solids. Appl Mech Rev. 50, 15-67 (1997). doi:10.1115/1.3101682

11. Baillie, RT: Long memory processes and fractional integration in econometrics. J Econ. 73, 5-59 (1996)

12. Magin, RL: Fractional calculus in bioengineering. Crit Rev Biomed Eng. 32, 1-377 (2004). doi:10.1615/CritRevBiomedEng. v32.10

13. Saadatmandi, A, Dehghan, M: A new operational matrix for solving fractional-order differential equations. Comput Math Appl. 59, 1326-1336 (2010). doi:10.1016/j.camwa.2009.07.006

14. Zhou, Y, Jiao, F, Li, J: Existence and uniqueness for $p$-type fractional neutral differential equations. Nonlinear Anal. 71, 2724-2733 (2009). doi:10.1016/j.na.2009.01.105

15. Galeone, L, Garrappa, R: Explicit methods for fractional differential equations and their stability properties. J Comput Appl Math. 228, 548-560 (2009). doi:10.1016/j.cam.2008.03.025

16. Trigeassou, JC, Maamri, N, Sabatier, J, Oustaloup, A: A Lyapunov approach to the stability of fractional differential equations. Signal Process. 91, 437-445 (2011). doi:10.1016/j.sigpro.2010.04.024

17. Deng, W: Smoothness and stability of the solutions for nonlinear fractional differential equations. Nonlinear Anal. 72 , 1768-1777 (2010). doi:10.1016/j.na.2009.09.018

18. Ghoreishi, F, Yazdani, S: An extension of the spectral Tau method for numerical solution of multi-order fractional differential equations with convergence analysis. Comput Math Appl. 61, 30-43 (2011). doi:10.1016/j.camwa.2010.10.027

19. Edwards, JT, Ford, NJ, Simpson, AC: The numerical solution of linear multi-term fractional differential equations: systems of equations. J Comput Appl Math. 148, 401-418 (2002). doi:10.1016/50377-0427(02)00558-7

20. Muslim, M: Existence and approximation of solutions to fractional differential equations. Math Comput Model. 49, 1164-1172 (2009). doi:10.1016/j.mcm.2008.07.013

21. Hardy, GH, Littlewood, JE, Pólya, G: Inequalities. Cambridge University Press, Cambridge, 2 (1988)

doi:10.1186/1687-1847-2012-33

Cite this article as: Chen: Oscillation criteria of fractional differential equations. Advances in Difference Equations 2012 2012:33.

\section{Submit your manuscript to a SpringerOpen ${ }^{\odot}$ journal and benefit from:}

- Convenient online submission

- Rigorous peer review

- Immediate publication on acceptance

- Open access: articles freely available online

- High visibility within the field

- Retaining the copyright to your article

Submit your next manuscript at $\boldsymbol{s p r i n g e r o p e n . c o m ~}$ 